\title{
Electrocardiographic Measurements of the QT Interval during Embryonic Development in Fertilized Chicken Eggs
}

\author{
Tanveer A Bhuiyan $^{1 *}$, Cristian Sevcencu ${ }^{1}$, Johannes J Struijk ${ }^{1}$, Jørgen K Kanters ${ }^{2}$, Claus Graff ${ }^{1}$ \\ ${ }^{1}$ Department of Health Science and Technology, Aalborg University, Aalborg Denmark \\ ${ }^{2}$ Laboratory of Experimental Cardiology, University of Copenhagen, Copenhagen, Denmark
}

\begin{abstract}
The fertilized chicken egg (embryonic chicken) shares the basic mechanism of electrophysiology and ionic currents with mammalian hearts. However, little is known about the cardiac repolarization process during embryo growth. We examined if the electrocardiographic $Q T$ interval was dependent on the stage of embryonic development.

ECGs were recorded from 4 fertilized chicken eggs for 6 days (day 13 to day 18). Eggs were kept in an incubator with a temperature of $37.4^{\circ} \mathrm{C}$. Chronic electrodes were implanted and a 5-minute ECG was recorded each day. Three measurements were made in noise free segments: Heart rate, QT interval, and the Fridericia corrected QT interval (QTCF), and an average value was calculated.

The QTcF interval decreased during embryo development. On day 18, QTCF was shorter than QTCF on day 13: $341 \mathrm{~ms}$ (95\% CI: $332 \mathrm{~ms}$ to $349 \mathrm{~ms}$ ) versus 417 (95\% CI: 325 ms to $509 \mathrm{~ms}$ ), $p=0.037$.

In fertilized chicken eggs, the repolarization process changes during development. This property makes the chicken embryo an interesting model for the study of drug effects on the QT interval at different stages of embryo development.
\end{abstract}

\section{Introduction}

The ionic basis of the chick embryonic action potential is similar to the most vertebrate hearts and studies of chick embryonic ion channels show that the permeability to $\mathrm{K}+$ increases during the embryonic period [1]. Potassium channels are responsible for the repolarization process and any change in $\mathrm{K}+$ permeability may have an effect on the electrocardiographic QT interval. Changes in ion channel permeability may also greatly affect the responsiveness of the myocardium to pharmacological agents.

In this study we have recorded in vivo electrocardiograms from fertilized chicken eggs to investigate the association between embryonic development and the duration of the QT interval.

\section{Methods}

\subsection{Study population}

ECGs were recorded from 4 fertilized chicken eggs for 6 days. Between recordings, the fertilized eggs were kept in an incubator with a temperature of $37.4^{\circ} \mathrm{C}$.

\subsection{Electrode implantation}

Gold plated chronic electrodes were used. The position of the embryo was confirmed prior to electrode placement by setting the egg against strong coherent light. The electrodes were cleaned in $80 \%$ alcohol solution to prevent bacterial formation. Three tiny holes were made with sterile needles and the electrodes were inserted at the equatorial region of the eggs $120^{\circ}$ apart from each other.

The two electrodes placed with the embryo in between them were connected to differential inputs and the third electrode was connected to the ground of the iWorx system (iWorx ${ }^{\mathrm{TM}}$ ).

After the implantation, the holes were immediately closed with acrylic cement and the egg was put back in the incubator.

\subsection{ECG recordings}

Continuous 5-minute ECGs were recorded from each egg on days: 13,14,15,16 and 18. A high pass filter (cutoff frequency $=1 \mathrm{~Hz}$ ) was used to remove baseline drift and a 15-tap moving average filter was used to smooth the ECG signal. Three measurements were made in noise free segments: Heart rate, QT interval, and the Fridericia corrected QT interval (QTcF), and an average value was calculated. A 10 second segment of a representative ECG recording is shown in figure 1. 

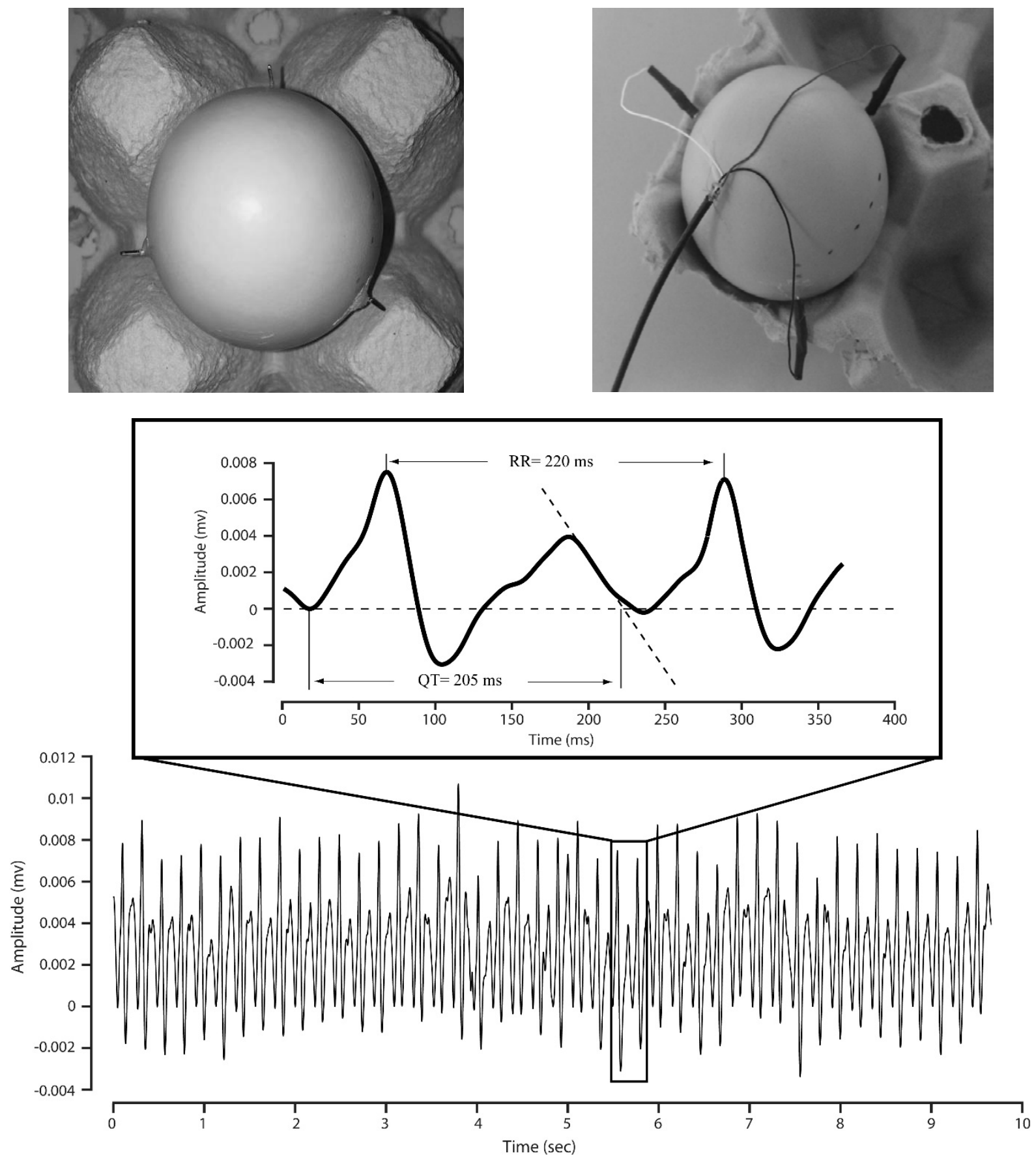

Figure 1: ECG recording from a fertilized chicken egg on day 18. Top left panel shows the position of implanted gold electrodes. Top right panel shows the electrodes connected to the lead cables. The middle panel illustrates the tangent method of QT interval measurement. Bottom panel shows a ten second excerpt of a 5-minute ECG.

\subsection{Statistical analysis}

Paired sample t-test was used to calculate the difference between QTcF on days 13 and 18. Statistical significance was defined as $\mathrm{p}<0.05$ and confidence intervals (CIs) were made at the $95 \%$ level.

\section{Results}

Figure 2 shows the gradual changes in heart rate, QT interval and QTcF during embryonic development from day 13 to day 18.

The heart rate increased from 215 bpm (95\% CI: 132 
bpm to $298 \mathrm{bpm}$ ) to $282 \mathrm{bpm}$ (95\% CI: $253 \mathrm{bpm}$ to 311 bpm).

The QT interval decreased from 275 ms (95\% CI: 175 $\mathrm{ms}$ to $376 \mathrm{~ms}$ ) to $210 \mathrm{~ms}$ ( $95 \% \mathrm{CI}: 181 \mathrm{~ms}$ to $240 \mathrm{~ms}$ ).

Mean QTcF gradually decreased from 417 ms (95\% CI: $325 \mathrm{~ms}$ to $509 \mathrm{~ms}$ ) to $341 \mathrm{~ms}$ (95\% CI: $332 \mathrm{~ms}$ to 349 $\mathrm{ms}), \mathrm{p}=0.037$.


Figure 2. ECG measurements on days 13 to 18 of embryonic development.

\section{Discussion}

To our knowledge, this is the first study to measure the QT interval during embryonic development in fertilized chicken eggs.

We observed a gradual decrease in QTcF from day 13 to day 18. Such shortening of the QT interval may be the result of increased expression during embryonic development of the genes encoding the cardiac potassium channels involved in repolarization. This is supported by a study of isolated chick myocardium where the magnitude of action potential prolongation by the $\mathrm{I}_{\mathrm{Kr}}$ blocker E4031 was found to be larger in 7-10 and 11-13 day old embryos compared to 14-20 day old embryos [2]. Moreover, the effect on action potential prolongation with the $\mathrm{I}_{\mathrm{Ks}}$ blocker chromanol 293B was generally small, suggesting that the $\mathrm{I}_{\mathrm{Kr}}$ current is primarily responsible for ventricular repolarization in the chick embryo and that $\mathrm{I}_{\mathrm{Kr}}$ changes gradually during embryonic development. As such, the chick embryo could potentially be used at various stages of development as a model for reduced repolarization reserve, which is also seen in patients with mutations in the hERG gene causing Long QT syndrome Type 2 by disrupting $\mathrm{I}_{\mathrm{Kr}}$.

The chick embryo may also be a suitable model for testing $\mathrm{I}_{\mathrm{Kr}}$ inhibiting drugs. In such studies, our findings of a varying QT interval show that it is important to take into account the marked changes that occur in the absence of drugs as the embryo develops.

\section{Conclusion}

In fertilized chicken eggs, the repolarization phase changes as the chick develops resulting in shortening of the QT interval.

\section{Acknowledgments}

The authors would like to thank Danhatch A/S for supplying the fertilized chicken eggs.

\section{References}

[1] Sperelakis N, Shigenobu K. Changes in membrane properties of chick embryonic hearts during development. The Journal of general physiology. 1972;60(4):430-53.

[2] Nouchi H, Kiyru N, Kimata $M$, et al. Developmental changes in action potential prolongation by $\mathrm{K}+$-channel blockers in chick myocardium. J Pharmacol Sci. 2011;115:235-8.

Address for correspondence.

Tanveer Ahmed Bhuiyan

Department of Health Science and Technology,

Aalborg University.

Frederik Bajers vej-7 C1-217, 9220 Aalborg, DK tb@hst.aau.dk 
